\title{
Severity Assessment of Complex and Repeated Intracranial Surgery in Rats
}

\author{
Ann-Kristin Riedesel Simeon O.A. Helgers Arif Abdulbaki \\ Gökce Hatipoglu Majernik Mesbah Alam Joachim K. Krauss Kerstin Schwabe \\ Department of Neurosurgery, Hannover Medical School, Hannover, Germany
}

\section{Keywords}

Neurosurgery · Animal well-being · Behavior - Refinement ·

Principal component analysis

\begin{abstract}
Introduction: Evidence-based grading of the impact of intracranial surgery on rat's well-being is important for ethical and legal reasons. We assessed the severity of complex and repeated intracranial surgery in a 6-hydroxydopamine (6OHDA) Parkinson's rat model with subsequent intracranial electrode implantation and in an intracranial tumor model with subsequent resection. Methods: Stereotactic surgery was performed in adult male rats with the same general anesthesia and perioperative pain management. In Parkinson's model, Sprague Dawley rats received unilateral injection of 6-OHDA $(n=11)$ or vehicle $(n=7)$ into the medial forebrain bundle as first operation (1st OP). After four weeks, neural electrodes were implanted in all rats as second operation (2nd OP). For tumor formation, BDIX/UlmHanZtm rats $(n=8)$ received frontocortical injection of BT4Ca cells as 1 st OP, followed by tumor resection as 2 nd OP after one week. Multiple measures severity assessment was done two days before and four days after surgery in all rats, compris-
\end{abstract}

ing clinical scoring, body weight, and detailed behavioral screening. To include a condition with a known burden, rats with intracranial tumors were additionally assessed up to a predefined humane endpoint that has previously been classified as "moderate". Results: After the 1st OP, only 6-OHDA injection resulted in transient elevated clinical scores, a mild long-lasting weight reduction, and motor disturbances. After the second surgery, body weight was transiently reduced in all groups. All other parameters showed variable results. Principal component analysis showed a separation from the preoperative state driven by motor-related parameters after 6-OHDA injection, while separation after electrode implantation and more clearly after tumor resection was driven by pain-related parameters, although not reaching the level of the humane endpoint of our tumor model. Conclusion: Overall, cranial surgery of different complexity only transiently and rather mildly affects rat's well-being. Multiple measures assessment allows the differentiation of model-related motor disturbances in Parkinson's model from potentially pain-related conditions after tumor resection and electrode implantation.

\section{(C) 2021 The Author(s)}

Published by S. Karger AG, Basel karger@karger.com www.karger.com/esr

Karger $\stackrel{\text { ' }}{5}$

GOPEN ACCESS
C 2021 The Author(s).

Published by S. Karger AG, Basel

This article is licensed under the Creative Commons Attribution 4.0 International License (CC BY) (http://www.karger.com/Services/ OpenAccessLicense). Usage, derivative works and distribution are permitted provided that proper credit is given to the author and the original publisher.
Correspondence to:

Ann-Kristin Riedesel, riedesel.ann-kristin@mh-hannover.de 


\section{Introduction}

A reliable, objective and standardized classification of severity in animal experimentation is the basis for a decisive harm-benefit analysis, essential for refinement, and required for high-quality research data. According to the EU Directive 2010/63, all experimental procedures must be categorized as to whether they induce a mild, moderate or severe condition of pain, distress, suffering or lasting harm. Together with laparotomy and thoracotomy, craniotomy under general anesthesia and appropriate analgesia are given as an example for a moderate condition. However, this classification does not take into account neurosurgery of different complexity or repeated neurosurgical interventions as applied in different rat models used in neuroscience research. Moreover, craniotomy describes a surgical operation in which a bone flap is temporarily removed from the skull to access the brain and which is only rarely used in neuroscience rodent models. Mostly, access to the brain during stereotactic surgery is performed via a small burr hole, often followed by microinjection of certain neuroactive compounds, or chronic implantation of medical devices with - depending on the actual model and brain region - different impact on the animal's condition.

Only few publications focus on the actual distress experienced by laboratory rats after neurosurgical procedures [1-5]. Body weight and clinical scoring are commonly used indicators of potential pain, suffering, or distress $[6,7]$. However, especially in neurological and neuropsychiatric rat models, the burden an animal experiences during procedures seldom consists of only one dimension, such as pain, but also includes model-specific disturbances and emotional components. Therefore, multiple measures assessment strategies have been proposed more recently, including species-specific behavior [8-12], home cage monitoring [13], as well as social- and motor behavior [3-5, 14-16].

Within the frame of the DFG research consortium "severity assessment in animal-based research", we tested the usefulness of multiple measures severity assessment schemes in the perioperative phase of intracranial surgery in neuroscience rat models routinely used in our laboratory: in Parkinson's model, unilateral intracranial injection of 6-hydroxydopamine (6-OHDA; group 1) or vehicle (group 2) as first operation (1st OP) for uniform degeneration of dopaminergic neurons in the substantia nigra and subsequent loss of dopamine in the projection region of the striatum. This was followed by implantation of neural electrodes as second operation (2nd OP) after four weeks. In the intracranial tumor model, intracranial injection of the BT4Ca-rat glioma cell line was done for intracranial tumor formation (group 3) as 1st OP, followed by tumor resection as 2 nd OP after one week. These models cover a wide range of intracranial neurosurgical approaches of potentially different severity levels, i.e., a small burr hole for stereotactic injection of vehicle, tumor cells or neurotoxic substances, intracranial implantation of electrodes chronically fixed to the rat's skull with anchor screws and a socket for cable connection, as well as a large craniotomy for tumor resection or implantation of cortical electrode grids for electrocorticogram recording. Using the intracranial tumor model also allows to compare the data of rats with intracranial tumor formation at the day of humane endpoint, which in our group is determined upon sudden mild to moderate deterioration of the clinical state and weight loss of 3-5 \% and was classified as "moderate" before [17].

\section{Materials and Methods}

\section{Animals and Housing Conditions}

All rats were part of ongoing scientific projects of our group. Twenty-six adult male rats ( $>250 \mathrm{~g}$ ) of two different rat strains (Sprague Dawley [SD] obtained from Charles River Laboratories, Germany, and BDIX/UlmHanZtm [BDIX] bred in the Central Animal Facility at Hannover Medical School) were used. Animals were pair-housed in standard Type IV open cages (1354G; Tecniplast, Hohenpeißenberg, Germany) in a ventilated cabinet (Classic scantainer; Scanbur, Karlslunde, Denmark; 20 air changes/h) with controlled temperature of $22 \pm 2{ }^{\circ} \mathrm{C}$, humidity of $55 \pm 10 \%$, and a light-dark cycle of $14 \mathrm{~h} / 10 \mathrm{~h}$ (light on at 6:00 a.m.). Only after electrode implantation, SD rats were single-housed in standard Type III open cages $(1291 \mathrm{H}$; Tecniplast) since later procedures in the ongoing research project required single housing of these rats. Rats were fed a standard diet (1324 TPF; Altromin, Lage, Germany) with free access to bottled water. Cage change was performed on a weekly basis with aspen wood ship bedding (AB P3; AsBe-wood, Gransee, Germany) and nesting material (Enviro-dri ${ }^{\circledR}$ coarse paper fibers; Claus, Berlin, Germany). The animals' health monitoring was performed according to FELASA recommendations using sentinel screening. Sample sizes were calculated for ongoing scientific projects, but not for the explorative approach of multiple measures severity assessment.

\section{Experimental Design}

The severity of neurosurgical interventions was assessed in the following experimental groups of neuroscience models. SD rats received local intracranial injection of 6-OHDA (group $1 ; n=11$ ) as model of Parkinson's disease or vehicle injection (group 2; $n=$ 7) as sham-control after simple randomization, followed by stereotactic implantation of electrodes in both groups four weeks later. In BDIX rats, the BT4Ca-rat glioma cell line was injected into the frontal cortex (FC) for tumor formation (group $3 ; n=8$ ), followed by tumor resection after one week, since at that time the tumor reliably develops into a size that can be easily resected [18]. See Figure 1a for an overview of groups and surgery. 


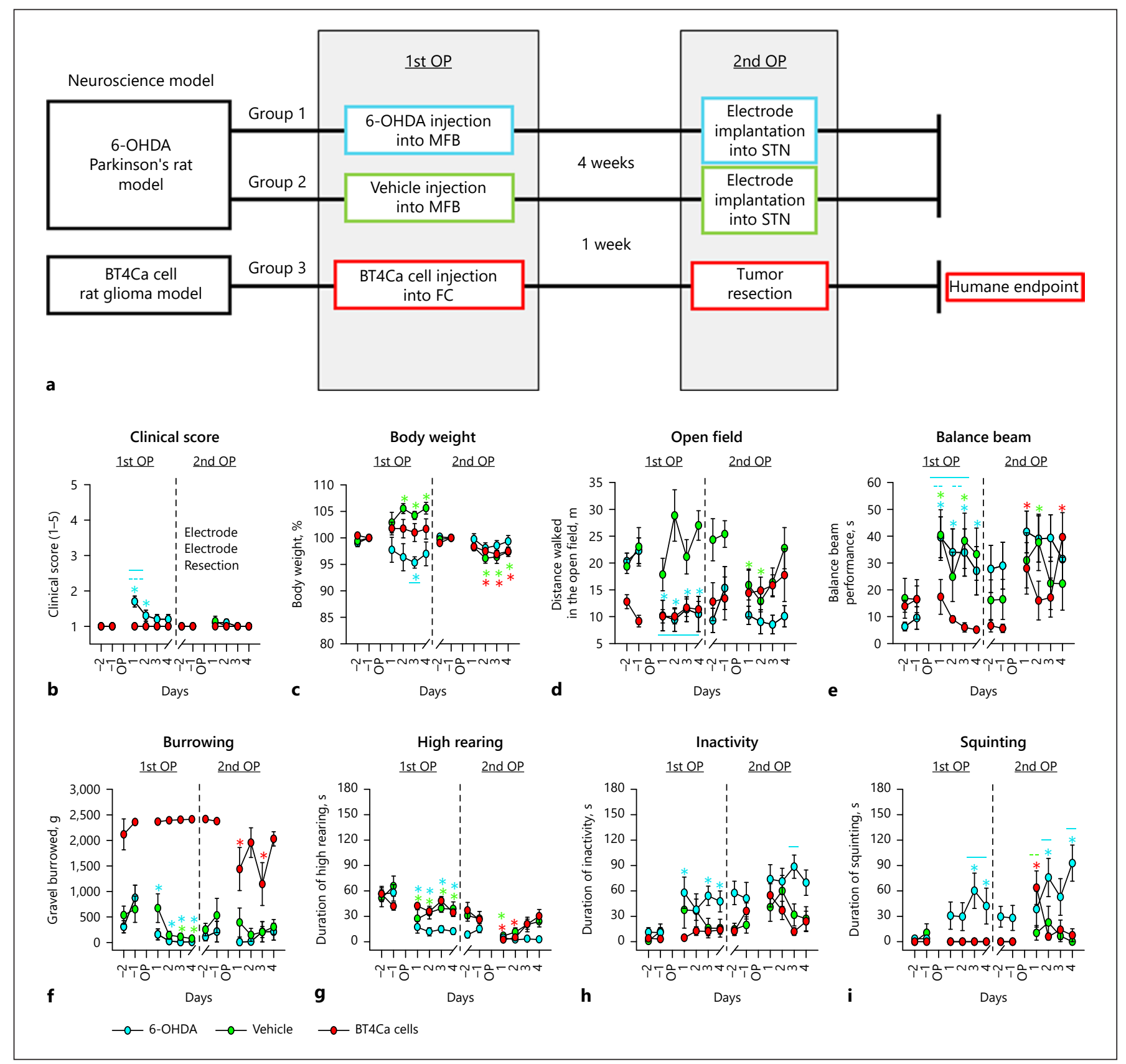

Fig. 1. Multiple measures severity assessment. a Experimental setup and overview of neuroscience models. 6-OHDA Parkinson's rat model with local intracranial injection of 6-OHDA for nigrostriatal loss of dopamine cells or vehicle as 1st OP, followed by stereotactic implantation of neural electrodes in both experimental groups as 2nd OP after four weeks. BT4Ca cell rat glioma model with intracranial injection of the BT4Ca-rat glioma cell line for intracranial tumor formation as 1st OP, followed by tumor resection as 2nd OP after one week. Clinical score (b), body weight (c), distance walked in the open field (d), balance beam performance (e), burrowing (f), as well as the duration of high rearing $(\mathbf{g})$, inactivity $(\mathbf{h})$, and squinting (i). Data are shown as mean \pm SEM two days before and four days after surgery. Asterisks $(*)$ indicate significant differences to the preop- erative measure within groups. Significant differences between groups are indicated by lines. A solid blue line stands for a significant difference between 6-OHDA- and vehicle-injected rats, a dashed blue line for a significant difference between 6-OHDA-injected and BT4Ca cell-injected rats, and a dashed green line between vehicleinjected and BT4Ca cell-injected rats. Two-way repeated measures ANOVA with factor group (6-OHDA/vehicle/cell injection [1st $\mathrm{OP}$ ], electrode implantation after 6-OHDA/vehicle injection, tumor resection [2nd OP] ) and day followed by a Bonferroni post-hoc test for group comparisons with $p<0.05$ indicating significant differences. 6-OHDA, 6-hydroxydopamine; 1st OP, first operation; 2nd $\mathrm{OP}$, second operation; ANOVA, analysis of variance; MFB, medial forebrain bundle; FC, frontal cortex; STN, subthalamic nucleus. 
At the end of the experiments, the animals were euthanized by an overdose of chloral hydrate $(720 \mathrm{mg} / \mathrm{kg})$. After breathing arrest and loss of reflexes, they were subjected to transcardial paraformaldehyde perfusion, followed by decapitation to remove the brain for subsequent histological analyses.

\section{Multiple Measures Severity Assessment}

The impact of intracranial interventions was assessed two days before and four days after surgery. In addition, the rats with intracranial tumors were assessed until the humane endpoint, which is defined in our group upon sudden mild to moderate deterioration of the clinical state and weight loss of 3-5\%, classified as "moderate" in previous work [17]. All rats were habituated and trained in the different test environments prior to actual testing. Tests were carried out in randomized order during the light phase of the daynight cycle, between 8:00 a.m. and 3:00 p.m.

\section{Clinical Scoring System and Body Weight Determination}

Animals were weighed each morning, and their general health condition was assessed according to the score adapted to Van Griensven et al. [19]: (1) physiological health condition with active, strong, and fast movements and normal exploratory behavior; (2) active with some interruptions of activity and exploratory behavior; (3) limited activity, exploratory behavior upon external stimuli, and intact muscle tone; (4) mild ataxia, no exploratory behavior, severely limited reaction to external stimuli, decreased muscle tone, hunched posture, ruffled fur, and delayed righting reflex; and (5) lethargic, no righting reflex, and premortal stage.

\section{Motor Activity}

Open Field

Each rat was placed individually in the black quadratic open field $(60 \times 60 \times 30 \mathrm{~cm})$ in a dimly lit room $(10-20 \mathrm{lux})$ and video recorded from above for $10 \mathrm{~min}$. The total distance moved was analyzed by the animal tracking system TopScan TM 1.0 (Clever Sys., Reston, VA, USA).

\section{Balance Beam Performance}

The balance beam was constructed of a quadratic wooden bar (length: $150 \mathrm{~cm}$, width: $1.8 \mathrm{~cm}$ ) installed $40 \mathrm{~cm}$ above the floor with a platform at one end. For testing, rats were placed in the middle of the beam and the time was measured until the rat reached the end platform. The home cage near the platform and rodent pellets (dustless precision pellets ${ }^{\circledR}$; Bio-Serv, Flemington, NJ, USA) placed on the platform were used as motivation. The test was aborted in case a rat fell off the beam or the maximum time limit of $60 \mathrm{~s}$ was reached.

\section{Species-Specific Behavior}

Nest Building

Nest building performance with $14 \mathrm{~g}$ of Enviro-dri ${ }^{\circledR}$ per rat placed in the cage once a week was assessed each morning (8:009:00 a.m.) using a score developed and adapted according to Van Loo and Baumans [20] and Schwabe et al. [21], i.e., (1) nesting material untouched or scattered in the cage; (2) nest flat; (3) nest slightly curved; and (4) nest deep similar to a bird's nest.

In case of pair housing, both rats were given the group score to allow statistical analysis as for other measure. After electrode implantation, rats were single-housed since later procedures in the ongoing research project required single housing of these rats and therefore each rat had a single score.

Severity Assessment of Intracranial Surgery
Burrowing Behavior

Tubes (length: $32 \mathrm{~cm}$, diameter: $10 \mathrm{~cm}$; entrance raised $6 \mathrm{~cm}$ above floor) were filled with $2.5 \mathrm{~kg}$ of gravel (size: $2-4 \mathrm{~mm}$ in diameter). For testing, rats were placed individually in an empty Type IV cage with a gravel-filled tube. After a total test time of 60 min, the weight of material left in the tube was measured and subtracted from the original tube weight at burrow start.

\section{Social Interaction}

Social interaction was tested by placing two cage partners together in the open field arena. The animals were filmed for five min, and social interaction was evaluated by a treatment-blinded person using the ODLogTM, Macropod software. Total social interaction included the duration (seconds) of playing, following, and sniffing at head or anogenital region.

\section{Video Analysis of Spontaneous Behaviors}

For assessment of spontaneous rat behavior, a rat ethogram described in detail by Roughan and Flecknell [22] and Thomas et al. [23] was used. Rats were placed in an empty Type III cage and video recorded for three min. The following parameters were evaluated manually and offline: back arching, fall/stagger, high rear, walking, inactive, head grooming, investigate, shake and squinting (partial closure of eyes).

\section{General Procedure for all Intracranial Surgeries}

For all surgical procedures, the rats were intraperitoneally anesthetized with chloral hydrate $(360 \mathrm{mg} / \mathrm{kg}, 10 \mathrm{~mL} / \mathrm{kg}$; Sigma-Aldrich, Darmstadt, Germany). For pain management, they were treated with carprofen $\left(50 \mathrm{mg} / \mathrm{mL}\right.$ Rimadyl ${ }^{\circledR}, 5 \mathrm{mg} / \mathrm{kg}$ s.c.; Zoetis, Berlin, Germany) intraoperatively and on the two following days. Additionally, the skin and periosteum were infiltrated with $1 \%$ xylocaine for local anesthesia (lidocainhydrochloride; AstraZeneca, Wedel, Germany). The anesthetized rats were secured in a stereotactic frame (Stoelting, Illinois, USA) with blunt ear bars, an incisivi bar, and the skin was incised. The periosteum was removed and, with reference to bregma, small trepanations (one $\mathrm{mm}$ in diameter) were drilled above targets depending on the coordinates given in the rat brain atlas of Paxinos and Watson [24]. After each surgery, the skin was closed by sutures (Ethilon ${ }^{\circledR}$ nylon suture 4-0) and the rats were placed on a warming mat until fully recovered from anesthesia.

\section{Rat Parkinson's Model}

Stereotaxic Injection of 6-OHDA (Group 1) or Vehicle

(Group 2) as 1st OP

Rats received stereotactic unilateral injection of 6-OHDA (3.6 $\mu \mathrm{g} / \mu \mathrm{L}$ in $0.02 \% \mathrm{~L}$-ascorbate-saline; Tocris Bioscience, Bristol, UK) at two sites of the right medial forebrain bundle, which results in uniform and complete degeneration of dopaminergic neurons in the substantia nigra and loss of dopamine in the projection region of the striatum. Sham-lesioned rats received vehicle solution $(0.02$ $\%$ L-ascorbate-saline). A 10- $\mu \mathrm{L}$ microsyringe (SEG Analytical Science Pty. Ltd., Victoria, Australia) was lowered through a burr hole (injection rate of $1 \mu \mathrm{L} / \mathrm{min}$; volume: $2.5 \mu \mathrm{L}$ and $3.0 \mu \mathrm{L}$, respectively) to the following coordinates relative to bregma: track one (1) anterior-posterior $(\mathrm{AP})=-4.0 \mathrm{~mm}$; lateral $(\mathrm{L})=-0.8 \mathrm{~mm}$; dorso$\operatorname{ventral}(\mathrm{V})=-8.0 \mathrm{~mm}$; and tooth bar set at $+3.4 \mathrm{~mm}$ and track two (2) $\mathrm{AP}=-4.4 \mathrm{~mm} ; \mathrm{L}=-1.2 \mathrm{~mm}$; dorsoventral $=-7.8 \mathrm{~mm}$; and tooth bar at $-2.3 \mathrm{~mm}$. After the injections, the cannula was left in place for three min to allow diffusion of the solution. 


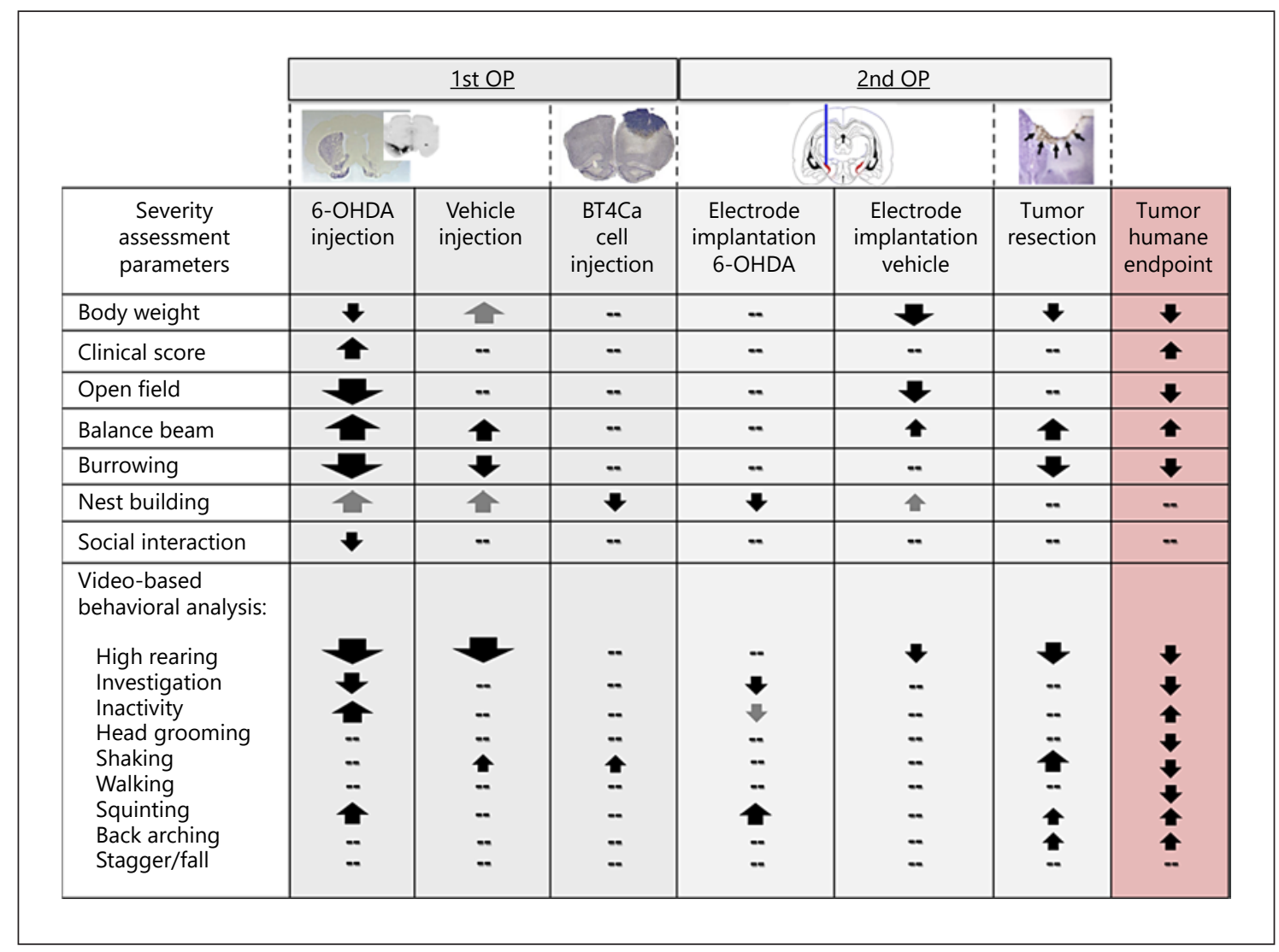

Fig. 2. Overview of multiple measures severity assessment. Arrows indicate significant alterations of the tested parameters from preoperative data. The size of the arrows symbolize the duration ( small arrow $=$ one day; big arrow $=$ two days; bigger arrow $=$ three days; biggest arrow $=$ four days), the direction an increase or decrease of a significant change. A grey arrow depicts an improvement. A dash represents no change of a parameter. Normalized body weight (\%), clinical score (1-5), open field (distance moved in meters), balance beam (run in seconds), species-specific behavior: burrowing (gravel in g burrowed out of a plastic tube), nest building with Enviro-dri ${ }^{\circledR}$ coarse paper fibers, total social interaction, and video-based behavioral analysis. Last column shows the data of the tumor rats at the day of humane endpoint. 1st OP, first operation; 2nd OP, second operation; 6-OHDA, 6-hydroxydopamine.
Stereotaxic Implantation of Electrodes for Recording and Stimulation as 2 nd OP

After four weeks, a 16 contacts microelectrode-array (parylene $C$ film metallized with gold $\left(3 \times 3\right.$ contacts; $3 \mathrm{~mm}^{2}$ surface area; $0.75 \mathrm{~mm}$ contact spacing; $100 \mu \mathrm{m}$ contacts diameter; and impedances of approximately $200 \mathrm{k} \Omega$; Blackrock Microsystems, Salt Lake City, UT, USA) was placed over the right motor cortex and sensorimotor cortex after fenestration of the cranial bone via a large craniotomy $(3 \mathrm{~mm} \times 3 \mathrm{~mm})$. The stereotactic coordinates for cortical surface area implantation were $\mathrm{AP}=+2$ to $-2 \mathrm{~mm}$; $\mathrm{L}$ $=-1$ to $4 \mathrm{~mm}$; and tooth bar at $-3.3 \mathrm{~mm}$. Additionally, a stimulation electrode (teflon-coated platinum-iridium electrode) was stereotactically implanted in the subthalamic nucleus through a small burr hole at the following coordinates relative to bregma and to the surface of the dura mater: $\mathrm{AP}=-3.8 \mathrm{~mm} ; \mathrm{L}=-2.5 \mathrm{~mm}$; and $\mathrm{V}=7.8 \mathrm{~mm}$. For reinforcement, four anchor screws $(1 \times 2$ mm; Hummer \& Rieß, Nürnberg, Germany) were wound to the skull and the electrodes fixed to the skull with dental acrylic ce- ment (Paladur ${ }^{\circledR}$; Kulzer, Hanau, Germany). Antibiotics were applied for eight days starting two days before surgery $(6.6 \mathrm{mg} / \mathrm{kg}$ s.c. marbofloxacin, Marbocyl ${ }^{\circledR}$ FD $1 \%$; Vétoquinol, Ravensburg, Germany).

\section{Rat Glioma Model}

Stereotaxic Injection of BT4Ca-Rat Cells (Group 3) as 1st OP

The BT4Ca-rat glioma cell line was stereotactically microinjected into the right frontal cortex of the rats through a small burr hole using a 10- $\mu \mathrm{L}$ microsyringe (SEG Analytical Science Pty. Ltd.; 10,000 BT4Ca cells/ $3 \mu \mathrm{L}$ PBS; injection rate: $1 \mu \mathrm{L} / \mathrm{min}$ ) as described in more detail before [18]. Bregma was used as reference point for the target coordinates: $\mathrm{AP}=+2.6 \mathrm{~mm}$ and $\mathrm{L}=+2.5 \mathrm{~mm}$. Injection took place at a depth of $2.8 \mathrm{~mm}$.

Stereotactic Tumor Resection as 2nd OP

One week after the injection of BT4Ca cells, the solid tumor was resected under microscopic view (Zeiss STEMI 2000-C; Zeiss, 
Oberkochen, Germany). The midline incision was reopened, and a craniotomy was made extending the original burr hole by two $\mathrm{mm}$ radially. After opening of the dura, the arachnoid was incised and the tumor was extracted using conventional microsurgical techniques. Hemostasis was achieved by rinsing with sterile $0.9 \%$ saline solution or by using Tabotamp ${ }^{\circledR}$ (Ethicon, Norderstedt, Germany) as described in Wu et al. [18].

\section{Statistical Analysis}

Statistical analysis was carried out with SigmaStat 4.0 and data visualization by SigmaPlot 14.0 (Systat Software, Erkrath, Germany). All data were presented as mean \pm SEM. Data were analyzed by two-way repeated measures analysis of variance (ANOVA) for either the 1st or the 2nd OP with experimental "group" as independent factor and "day" (two days before and four days after OP) as repeated within group factor. For the 1st OP, the 6-OHDA injection ("6-OHDA") of Parkinson's model was compared to sham vehicle injection and cell injection of the tumor model ("BT4Ca cells"). For the 2nd OP, electrode implantation after 6-OHDA and vehicle injection ("Electrode") in Parkinson's model was compared to tumor resection ("Resection"). Bonferroni post-hoc testing against the day before OP was conducted in case of significant ANOVA. All tests were performed two-sided with a level of significance set to a $p$ value $<0.05$. All data are summarized in Figure 2. Moreover, data of clinical score, weight, motor activity (open field and balance beam), as well as examples for species-specific behavior (burrowing), and video analysis of spontaneous behavior (high rearing, inactivity, and squinting) are shown as mean \pm SEM for different groups and days.

Additionally, all measures were subjected to principal component analysis (PCA) using the factoextra package [25] in base $\mathrm{R}$ [26]. The principal components of the first two dimensions for all days were plotted, as well as the factor loadings and contributions (Fig. 3).

\section{Results}

The data of one rat that died during surgery for electrode implantation were completely excluded from analysis, leaving $n=10$ for 6-OHDA lesion, $n=7$ for sham lesion and subsequent electrode implantation, and $n=8$ for intracranial tumor and subsequent resection.

\section{Clinical Scoring and Body Weight}

\section{Clinical Scoring}

After the 1st OP, only 6-OHDA injection led to a temporarily elevated score on the first two postoperative days (not exceeding score of 2) both compared to the preoperative measures, as well as to the vehicle- and tumor cellinjected groups (post-hoc testing $p<0.05$ after significant ANOVA for all factors [all $F$ values $>4.984$ and all $p$ values $<0.007]$ ). For the 2nd OP, ANOVA showed no significant effect for either factor (Fig. 1b, 2).

Severity Assessment of Intracranial Surgery

\section{Body Weight}

After 1st OP, only rats with 6-OHDA lesions lost weight on postoperative day three and compared to vehicle-injected rats on all postoperative days (post-hoc testing $p<0.05$ after significant ANOVA for the factor group $\left[F_{2,110}=5.744 ; p=0.010\right]$ and interaction between the factor group and day $\left.\left[F_{10,110}=3.819 ; p<0.001\right]\right)$. Interestingly, vehicle-injected rats even gained weight after surgery. For the 2nd OP, body weight was mildly reduced after electrode implantation in vehicle-injected rats on day two to four and after tumor resection on day two to four compared to the preoperative measure (post-hoc testing $p<0.05$ after significant ANOVA for the factor day $\left.\left[F_{5,110}=12.100 ; p<0.001\right]\right)$. No significant group differences were noted (Fig. 1c, 2).

\section{Motor Activity}

\section{Open Field Performance}

Before surgery, BDIX rats already covered less distance in the open field than SD rats. After the 1st OP, in 6-OHDA-injected rats the distance walked significantly decreased for all days, both compared to preoperative measure and to vehicle-injected rats (post-hoc $p<0.05$ after significant ANOVA for all factors [all $F$ values $>$ 2.673 and all $p$ values $<0.025]$ ). For the 2 nd OP, only electrode implantation in the vehicle group led to reduced locomotion on the first and second postoperative day (all $p$ values $<0.05$ after significant ANOVA for all factors [all $F$ values $>1.932$ and all $p$ values $>0.048$; Fig. 1 d, 2]).

\section{Balance Beam Performance}

After the 1st OP, the time to reach the end platform was extended in SD rats after 6-OHDA injection for all days, and after vehicle injection on days one and three compared to the preoperative measure and to BDIX rats after cell injection (post-hoc $p<0.05$ after significant ANOVA for the factor day $\left[F_{5,110}=8.039 ; p<0.001\right]$ and interaction between factor day and group $\left.\left[F_{10,110}=4.082 ; p<0.001\right]\right)$. After the 2nd OP, vehicle-injected rats undergoing electrode implantation had a prolonged walking time on the second postoperative day and rats with tumor resection on the first and fourth postoperative day compared to the preoperative measure $(p<0.05$ after significant ANOVA for the factor day $\left[F_{5,110}=6.559 ; p<0.001\right]$ and interaction between factor day and group $\left[F_{10,110}=2.098 ; p=0.030\right.$; Fig. 1e, 2$]$ ).

\section{Species-Specific Behavior}

Burrowing

BDIX rats always burrowed more gravel than SD rats, which either did not engage at all or only burrowed small 


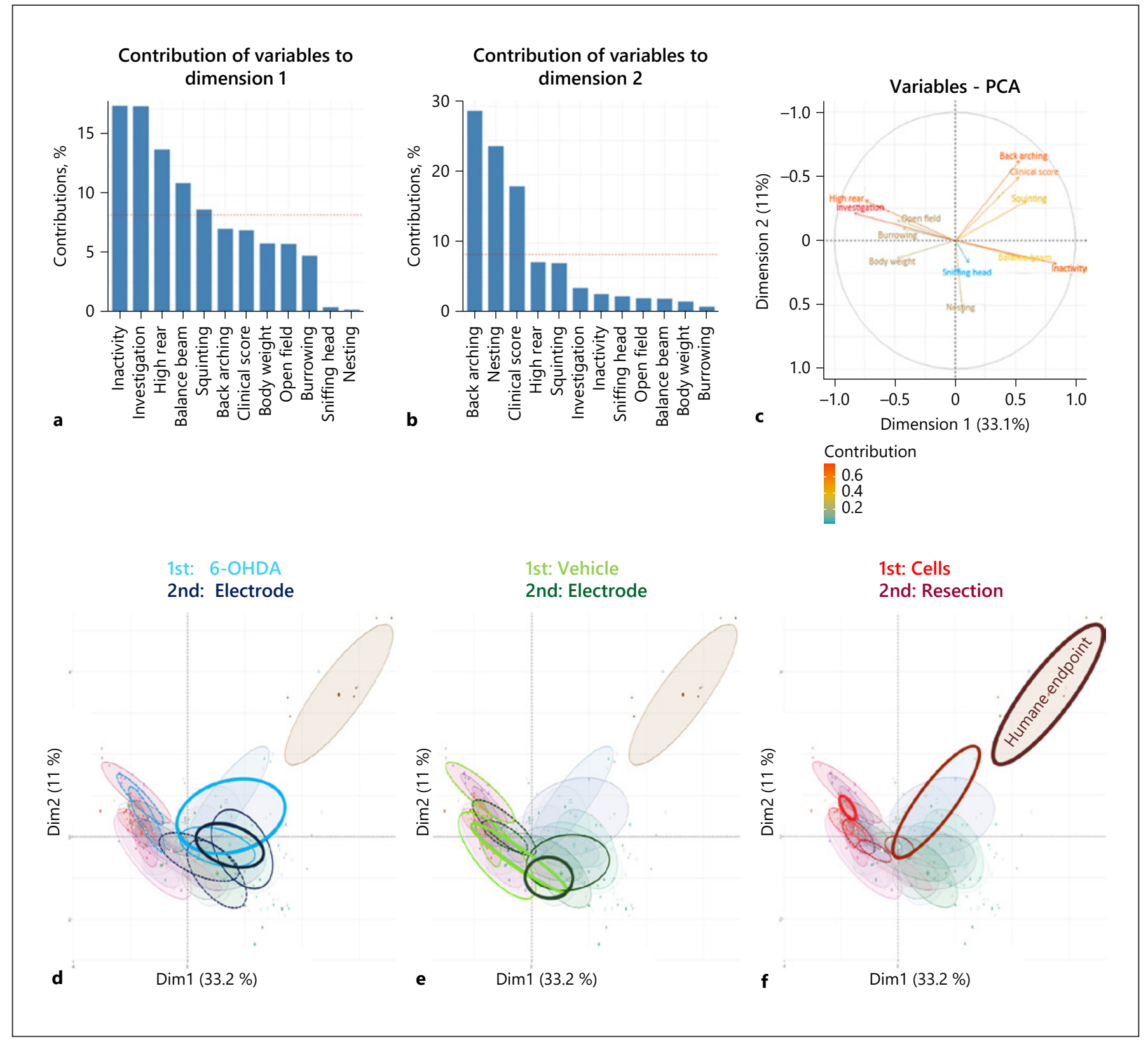

Fig. 3. PCA. a-f PCA of the parameters tested. The contribution of variables (inactivity, investigation, high rear, balance beam, squinting, back arching, clinical scoring, body weight, open field, burrowing, sniffing head as part of social interaction, and nesting) to dimension-1 (a) and dimension-2 (b) are shown in percent. The red dashed line indicates the threshold for the equal contribution of each parameter. Illustration of the first 2 dimensions of the PCA is shown as arrows (c) with $\operatorname{Dim} 1(33.2 \%)$ on the $\mathrm{x}$-axis and Dim2 (11\%) on the $y$-axis. Length, direction, and color of the arrows code for level of contribution of each parameter to the different dimensions. $\mathbf{d}-\mathbf{f}$ Clusters are based on the PCA for each day and surgery. Ninety-five percent confidence ellipses around group clusters are shown as different colors and symbols for each day. For each group (6-OHDA, vehicle, and tumor), corresponding clusters before (dashed lines) and after surgery (solid line; only the first two days after surgery are highlighted with the first postoperative day indicated by a thick solid line; after that, clusters go back to the preoperative measure) are highlighted in colors referring to Figure 1. Light brown circle stands for data of rats with intracranial tumor formation at the day of humane endpoint determination with a known burden that has previously been classified as "moderate" [17]. PCA, principal component analysis; 6-OHDA, 6-hydroxydopamine. 
amounts. After the 1st OP, 6-OHDA injection in SD rats led to reduced burrowing on all postoperative days and vehicle injection in SD rats on postoperative day three and four as compared to the preoperative measure (posthoc $p<0.05$ after significant ANOVA for all factors [all $F$ values $>3.270$ and all $p$ values $<0.001]$ ). After the 2 nd $\mathrm{OP}$, only tumor resection led to reduced burrowing on the first and third postoperative days as compared to the preoperative measure (post-hoc $p<0.05$ after significant ANOVA for all factors [all $F$ values $>2.464$ and all $p$ values $<0.011$; Fig. 1f, 2]).

\section{Nest Building}

Nesting performance varied throughout the assessment without obvious relation to surgery and was even enhanced at some postoperative days (see Fig. 2). For the 1 st OP, only on the day after cell injection nesting scores significantly declined as compared to the preoperative measure (post-hoc $p<0.05$ after significant ANOVA for the factor interaction $\left[F_{5,110}=6.598 ; p<0.001\right]$ and interaction between factor day and group $\left[F_{10,110}=4.143\right.$; $p<$ $0.001])$. Interestingly, after 6-OHDA and vehicle-injection nesting even improved after surgery. For the 2nd OP, nesting scores significantly declined on the day after electrode implantation in 6-OHDA-lesioned rats as compared to the preoperative measure and compared to vehicle injection on the first postoperative day (post-hoc $p<0.05$ after significant ANOVA for the factor interaction between factor day and group $\left[F_{10,110}=3.680 ; p<\right.$ $0.001])$. Interestingly, after electrode implantation in vehicle-injected rats, nesting even improved (Fig. 2).

\section{Social Interaction}

After the 1st OP, 6-OHDA-injected rats spent less time interacting with the social partner as compared to the preoperative measure on the first postoperative day and compared to vehicle-injected rats on the first, third, and fourth postoperative day (post-hoc $p<0.05$ after significant ANOVA for all factors [all $F$ values $>2.009$ and all $p$ values $<0.039]$ ). Social behavior of BDIX rats was less intense than that of SD rats, but not affected by either surgery. For the 2nd OP, ANOVA showed no significant effect for either factor (Fig. 2).

\section{Video-Based Behavioral Analysis}

Only the behaviors that were significantly affected by surgery are described in detail here. Overall, spontaneous rat behavior was mostly affected after 6-OHDA injection as 1 st $\mathrm{OP}$ and tumor resection as 2 nd OP.

\section{High Rearing}

After the 1st OP, high rearing was reduced in 6-OHDAand vehicle-injected rats on all four postoperative days compared to the preoperative measure (post-hoc $p<0.05$ after significant ANOVA for all factors [all $F$ values $>$ 3.765 and all $p$ values $<0.037]$ ). After the 2 nd OP, high rearing was decreased two days after tumor resection and on the first postoperative day after electrode implantation in vehicle-injected rats as compared to the preoperative measure (post-hoc $p<0.05$ after significant ANOVA for all factors [all $F$ values $>2.010$ and all $p$ values $<0.039$; Fig. 1g, 2]).

\section{Inactivity}

After the 1st OP, inactivity increased in 6-OHDA-injected rats on the first, third, and fourth postoperative day compared to the preoperative measure (post-hoc $p<0.05$ after significant ANOVA for all factors [all $F$ values $>$ 3.679 and all $p$ values $<0.042]$ ). After the 2 nd OP, inactivity increased in 6-OHDA-injected rats undergoing electrode implantation on the third postoperative day compared to vehicle-injected rats undergoing electrode implantation (post-hoc $p<0.05$ after significant ANOVA for the factor group $\left[F_{22,110}=5.931 ; p=0.009\right]$ and day $\left[F_{5,110}=2.859 ; p=0.018\right]$; Fig. 1h, 2]).

\section{Squinting}

After the 1st OP, squinting increased in 6-OHDA-injected rats compared to the preoperative measure and compared to vehicle-injected rats on postoperative days three and four (post-hoc $p<0.05$ after significant ANOVA for the factor day $\left[F_{22,110}=5.685 ; p=0.010\right]$ ). After the 2nd OP, squinting increased in 6-OHDA-injected rats with electrode implantation on postoperative days two and four and BDIX rats with tumor resection on the first postoperative day as compared to the preoperative measure and to vehicle-injected rats with electrode implantation (post-hoc $p<0.05$ after significant ANOVA for all factors [all $F$ values $>3.123$ and all $p$ values $<0.011$; Fig. 1i, 2]).

\section{Back Arching}

After the 1st OP, ANOVA showed no significant effect for either factor. After the 2nd OP, rats with tumor resection displayed more back arching behavior on the first postoperative day as compared to the preoperative measure and to vehicle-injected rats with electrode implantation (post-hoc $p<0.05$ after significant ANOVA for the factor interaction $\left[F_{10,110}=2.496 ; p=0.010\right.$; Fig. 2]). 
Investigation

After the 1st OP, rats with 6-OHDA injection spent less time investigating their environment on the first and third postoperative days compared to the preoperative measure (post-hoc $p<0.05$ after significant ANOVA for the factor group $\left[F_{22,110}=4.099 ; p=0.031\right]$ and day $\left[F_{5,110}=3.372\right.$; $p=0.007])$. After the 2nd OP, rats with 6-OHDA injection undergoing electrode implantation spent less time investigating their environment on the third postoperative day compared to the preoperative measure (post-hoc $p<0.05$ after significant ANOVA for the factor day $\left[F_{5,110}=4.131\right.$; $p=0.002]$ and interaction between factor group and day $\left[F_{10,110}=2.020 ; p=0.038 ;\right.$ Fig. 2$\left.]\right)$.

\section{Principal Component Analysis}

PCA was conducted in order to better depict the importance and usefulness of our significant individual test results. PCA identified inactivity, investigation, high rearing, balance beam, and squinting as most meaningful parameters for the first principle component (Dim 1; Fig. 3a). Factors most contributing to the second principal component included back arching, nest building, and clinical scoring (Dim 2; Fig. 3b). The first 2 principal components account for $44.1 \%$ of the total data variance (Dim 1: $33.2 \%$; Dim 2: $11 \%$; Fig. 3c).

Based on the first two dimensions of the PCA, confidence ellipses (95\%) around group clusters for each day and surgery were created (Fig. 3d-f). A clear separation between the experimental groups could be seen after the 6-OHDA injection, which lasted until the second surgery. Electrode implantation in these rats had no further effect (Fig. 3d). In addition, both vehicle and cell injection as first surgery had no separating effect as compared to clusters before surgery, whereas electrode implantation as second surgery led to a partial (Fig. 3e) and tumor resection to a full separation (Fig. 3f). To further evaluate the severity status of our surgeries, we added the data of rats with intracranial tumor formation at the day of humane endpoint determination, i.e., sacrifice upon sudden deterioration of the clinical state and weight loss of 3-5\%, which was classified as "moderate" [27]. This state showed the most prominent separation from all other surgical procedures (light brown circle; Fig. 3d-f).

\section{Discussion}

A valid procedure to determine the degree of pain, distress, and suffering experienced by an animal, also taking into account model-specific aspects, is difficult. As part of a DFG research consortium addressing severity assessment strategies in animal-based research, we apply and validate existing methods to establish objective and standardized procedures for this purpose in neuroscience models [28]. Our study showed that with the perioperative pain management applied the severity of different neurosurgical approaches was rather mild, depending on the complexity of neurosurgery and model-specific aspects.

After 6-OHDA injection through a small burr hole into the medial forebrain bundle for nigrostriatal dopamine lesioning, clinical scores were only mildly and transiently elevated on the first two postoperative days, together with a mild weight reduction that thereafter increased in parallel to vehicle-injected rats. As expected in a model for Parkinson's disease, behaviors attributed to the motor domain (distance traveled in the open field and balance beam performance) were chronically reduced. In contrast, vehicle injection or injection of tumor cells through a small burr hole into the FC had no effects on all parameters tested. In this regard, it has been repeatedly reported before by other groups that even large lesions in the FC of rats are well tolerated [29].

However, the second surgery (electrode implantation and tumor resection) resulted in a transient mild but significant weight loss of 3-4 \% in almost all groups, but not in elevated clinical scores. Even though the second surgery was more complex also involving large craniotomies, it should be recognized that electrode implantation and tumor resection were done in adult rats. At this age, the weight had already reached a steady state, whereas the first surgery was done in developing "weight-gaining" rats, which may have masked weight loss upon surgery. Notably, body weight change is an easily obtainable and objective parameter, which correlates with severity conditions in various models. Nevertheless, experiment and model-specific considerations are necessary for the rational integration of this parameter in severity assessment schemes [30]. Furthermore, while clinical scoring reliably identifies moderate or severely affected rats, it can hardly be used to detect a mild impairment as shown in different other studies by a high inter-rater variability and low sensitivity $[27,31,32]$.

Interestingly, although natural, innate rodent behavior like burrowing, nest building, and social interaction have recently been proposed to be useful for the assessment of animal well-being [3, 4, 9-12, 33, 34], these measures were only marginally or not affected by either intracranial surgery. Similar findings have been described before, both with regard to burrowing [35] and to nesting 
[21]. Moreover, data varied extensively within groups and differences were mainly related to the two different rat strains. Detailed video-based behavioral analysis, however, showed that squinting, rearing, exploratory behavior, inactivity, and back arching were affected at least to some extent, but without obvious pattern with regard to the different intracranial approaches tested.

Since separate analysis of the different testing procedures did not show a conclusive pattern attributed to the intracranial approaches, we performed a PCA comprising all parameters that were significantly affected. This analysis identified rearing, back arching, exploratory behavior, and inactivity as most valuable parameters, followed by clinical scoring, balance beam, and squinting. The injection of 6-OHDA resulted in a clear and longlasting separation from preoperative measurements, which can be mainly explained by motor-related disturbances as expected in a chronic model for Parkinson's disease. Instead, both vehicle and cell injection as 1st OP had no separating effect as compared to preoperative measurements, whereas electrode implantation as 2 nd OP led to a partial, and tumor resection to a full separation of this group at least on the first day after surgery. Interestingly, this separation was mostly due to squinting and back arching, which are considered to be pain-related $[13,36-39]$. On the following three days, clusters go back to the normal state before operation, although analgesia was only given for the first two postoperative days.

With PCA, we identified a clear effect of the 6-OHDA injection and intracranial tumor resection, but the question of how "severe" these approaches should be classified still remained. To address this question, we included the data of the rats with intracranial tumor formation at the day of humane endpoint determination, i.e., sacrifice upon sudden weight loss of 3-5\% together with a deteriorated clinical state of mild to moderate, which has been classified as "moderate" in previous work of our group [17]. The prominent separation of this endpoint state from all other surgical procedures indicates that even tumor resection, which involves a large craniotomy and removal of voluminous tumor tissue, or electrode implantation with several screws wound to the skull, rather mildly affects rat's well-being. From a clinical point of view, the mild effects seen after intracranial surgery corroborate observations in patients where certain circumstances require surgery in awake patients with only local anesthesia, e.g., implantation of electrodes for deep brain stimulation [40-42].

Parameters identified as most informative by PCA were achieved from video-based behavioral observations of individual rats. Although aspects of this evaluation are also typical components of the clinical score (rearing as part of exploratory behavior and back arching, also described as hunched position), or also measured in the open field (distance moved or inactivity), they did not highlight when statistically analyzed as single parameters. One reason could be that rats as prey animals try to mask any signs of pain, distress, or discomfort in the presence of an experimenter [22] or in a foreign environment [43]. Moreover, clinical scoring is brief and influenced by the examiner's subjective point of view. An automated videobased behavioral monitoring system focusing on specific behavioral components in the home cage may be advantageous with that regard. It has been proposed before that postoperative pain-related behavior can be reliably detected by such analysis, preferably when filmed at night under red light $[22,44]$. Such a video-based analysis system may also include squinting as pain-related parameter and part of the grimace scale.

Although behavioral analysis is a relatively easy and inexpensive key method used in the assessment of welfare [45], data analysis is very time-consuming and does not allow prompt assessment of the animal's state [44, 46]. Data interpretation is often ambiguous despite high standardization of protocols and highly skilled experimenters [47]. The assessment parameters described here are therefore mainly helpful for the characterization of new neurosurgical approaches but do not offer a practical and useful routine system for assessing the severity of procedures. Notably, these analyses are also not suitable for severity assessment in individual rats. A rat-adapted "Relative Severity Assessment Score (RELSA)" may be valuable in identifying individual rats that are more severely affected by surgery [48].

\section{Conclusion}

Complex or repeated intracranial operations transiently and generally mildly affect rat's well-being at least with the perioperative pain management applied in this study. Only the 6-OHDA injection results in a slight but chronically reduced weight loss together with disturbed motorrelated function, as can be expected in a rat model of Parkinson's disease. Multiple measures assessment including PCA allows the differentiation of model-related motor disturbances in Parkinson's model from potentially painrelated conditions after tumor resection and electrode implantation. The identified "key behaviors" may be useful for characterizing new neurosurgical interventions with 
regard to the assessment of pain and distress, but also as a basis for developing and implementing a standardized and automated video-based analysis system.

\section{Acknowledgment}

We would like to thank Monika van Iterson for her excellent support and analysis of the social interaction videos.

\section{Statement of Ethics}

The experiments (intracranial glioma model in BDIX rats: license number AZ 33.12-42502-04-14/1533 and Parkinson's model in SD rats: license number AZ 33.8-42502-04-16/2315) were approved by the local Animal Ethics Committee (Lower Saxony State Office for Consumer Protection and Food Safety, LAVES). All investigations complied with the EU directive 2010/63 and the German Animal Welfare Act. All procedures and reporting were performed according to the ARRIVE guidelines including the 3R concept. All efforts were made to minimize the number of animals used and their suffering.

\section{Conflict of Interest Statement}

The authors have no conflicts of interest to declare.

\section{Funding Sources}

This work was supported by Grants of the German Research Foundation [DFG research group FOR 2591, GZ: SCHW1176/71], which were used to cover costs for the experimental studies described in the manuscript. The funding body was not involved in the design of the study, collection, analysis, and interpretation of data and in writing the manuscript.

\section{Author Contributions}

Concept, design, and funding acquisition were contributed by K.S. Supervision was contributed by K.S. and A.-K.R. Acquisition was contributed by A.-K.R., G.H.M., S.O.A.H., A.A., and M.A. Statistical analysis and interpretation of data were contributed by A.K.R. and S.O.A.H. Drafting of the manuscript was contributed by A.-K.R. Revision of the manuscript was contributed by A.-K.R., S.O.A.H., K.S., and J.K.K. All authors provided critical feedback and helped shape the research, analysis, and manuscript and approved the final version to be published.

\section{Data Availability Statement}

All data generated or analyzed during this study are included in this article. Further enquiries can be directed to the corresponding author.

\section{References}

1 Pinkernell S, Becker K, Lindauer U. Severity assessment and scoring for neurosurgical models in rodents. Lab Anim. 2016;50(6): $442-52$.

2 Marschner L, Wogensen E, Mogensen J, Abelson K. Implementation of a functional observation battery for the assessment of postoperative well-being in rats subjected to fimbria-fornix transection. In Vivo. 2016 Mar-Apr;30(2):77-82.

3 Seiffert I, van Dijk RM, Koska I, Di Liberto V, Möller C, Palme R, et al. Toward evidencebased severity assessment in rat models with repeated seizures: III. Electrical post-status epilepticus model. Epilepsia. 2019;60(8): 1539-51.

4 Möller C, Wolf F, van Dijk RM, Di Liberto V, Russmann V, Keck M, et al. Toward evidencebased severity assessment in rat models with repeated seizures: I. Electrical kindling. Epilepsia. 2018;59(4):765-77.

5 Koska I, van Dijk RM, Seiffert I, Di Liberto V, Möller C, Palme R, et al. Toward evidencebased severity assessment in rat models with repeated seizures: II. Chemical post-status epilepticus model. Epilepsia. 2019;60(10): 2114-27.
6 Morton DB, Griffiths PH. Guidelines on the recognition of pain, distress and discomfort in experimental animals and an hypothesis for assessment. Vet Rec. 1985;116(16):431-6.

7 Bugnon P, Heimann M, Thallmair M. What the literature tells us about score sheet design. Lab Anim. 2016;50(6):414-7.

8 Hohlbaum K, Bert B, Dietze S, Palme R, Fink $\mathrm{H}$, Thöne-Reineke C. Impact of repeated anesthesia with ketamine and xylazine on the well-being of C57BL/6JRj mice. PLoS One. 2018;13(9):e0203559.

9 Jirkof P. Burrowing and nest building behavior as indicators of well-being in mice. J Neurosci Methods. 2014;234:139-46.

10 Deacon RM. Burrowing in rodents: a sensitive method for detecting behavioral dysfunction. Nat Protoc. 2006;1(1):118-21.

11 Andrews N, Legg E, Lisak D, Issop Y, Richardson D, Harper S, et al. Spontaneous burrowing behaviour in the rat is reduced by peripheral nerve injury or inflammation associated pain. Eur J Pain. 2012;4:485-95.
12 Wodarski R, Morland R, Huang W, Rice ASC Ligocki M, Delaney A, et al. Cross-centre replication of suppressed burrowing behaviour as an ethologically relevant pain outcome measure in the rat: a prospective multicentre study. Pain. 2016;157(10):2350-65.

13 Roughan JV, Flecknell PA. Behavioural effects of laparotomy and analgesic effects of ketoprofen and carprofen in rats. Pain. 2001; 90(1-2):65-74

14 Combs DJ, D’Alecy LG. Motor performance in rats exposed to severe forebrain ischemia: effect of fasting and 1,3-butanediol. Stroke. 1987;18(2):503-11.

15 Craft TKS, Glasper ER, McCullough L, Zhang $\mathrm{N}$, Sugo N, Otsuka T, et al. Social interaction improves experimental stroke outcome. Stroke. 2005;36(9):2006-11.

16 Johansson BB, Ohlsson AL. Environment, social interaction, and physical activity as determinants of functional outcome after cerebral infarction in the rat. Exp Neurol. 1996;139(2): 322-7.

17 Helgers SOA, Talbot SR, Riedesel A-K, Wassermann L, Wu Z, Krauss JK, et al. Body weight algorithm predicts humane endpoint in an intracranial rat glioma model. Sci Rep. 2020;10(1):9020. 
$18 \mathrm{Wu}$ Z, Nakamura M, Krauss JK, Schwabe K, John N. Intracranial rat glioma model for tumor resection and local treatment. J Neurosci Methods. 2018;299:1-7.

19 Van Griensven M, Dahlweid FM, Giannoudis PV, Wittwer T, Böttcher F, Breddin M, et al. Dehydroepiandrosterone (DHEA) modulates the activity and the expression of lymphocyte subpopulations induced by cecal ligation and puncture. Shock. 2002;18(5):4459.

20 Van Loo PL, Baumans V. The importance of learning young: the use of nesting material in laboratory rats. Lab Anim. 2004;38(1):17-24.

21 Schwabe K, Boldt L, Bleich A, van Dijk RM, Helgers SOA, Häger C, et al. Nest-building performance in rats: impact of vendor, experience, and sex. Lab Anim. 2020;54(1):17-25.

22 Roughan JV, Flecknell PA. Behavioural effects of laparotomy and analgesic effects of ketoprofen and carprofen in rats. Pain. 2001; 90(1-2):65-74.

23 Thomas A, Miller A, Roughan J, Malik A, Haylor K, Sandersen C, et al. Efficacy of intrathecal morphine in a model of surgical pain in rats. PLoS One. 2016;11(10):e0163909.

24 Paxinos G, Watson C. The rat brain in stereotaxic coordinates. 4 th ed. San Diego: Academic Press; 1998.

25 Kassambara A, Mundt F. Factoextra: extract and visualize the results of multivariate data analyses (1.0.4 ed.). R package CRAN. 2019 [cited 2020 Mar 23]. Available from: http:// www.sthda.com/english/rpkgs/factoextra.

$26 \mathrm{R}$ Core Team. R: a language and environment for statistical computing. $\mathrm{R}$ foundation for statistical computing. 2021 [cited 2020 Mar 23]. Available from: http://www.R-project. org/.

27 Arras M, Rettich A, Cinelli P, Kasermann HP, Burki K. Assessment of post-laparotomy pain in laboratory mice by telemetric recording of heart rate and heart rate variability. BMC Vet Res. 2007;3:16.
28 Bleich A, Tolba RH. How can we assess their suffering? German research consortium aims at defining a severity assessment framework for laboratory animals. Lab Anim. 2017;51(6): 667.

29 Kolb B, Petrie B, Cioe J. Recovery from early cortical damage in rats. VII. Comparison of the behavioural and anatomical effects of medial prefrontal lesions at different ages of neural maturation. Behav Brain Res. 1996;79(12):1-14.

30 Talbot SR, Biernot S, Bleich A, van Dijk RM, Ernst L, Häger C, et al. Defining body-weight reduction as a humane endpoint: a critical appraisal. Lab Anim. 2020;54(1):99-110.

31 Palle P, Ferreira FM, Methner A, Buch T. The more the merrier? Scoring, statistics and animal welfare in experimental autoimmune encephalomyelitis. Lab Anim. 2016;50(6):42732 .

32 Keubler LM, Hoppe N, Potschka H, Talbot SR, Vollmar B, Zechner D, et al. Where are we heading? Challenges in evidence-based severity assessment. Lab Anim. 2020;54(1):50-62.

33 Jirkof P, Cesarovic N, Rettich A, Nicholls F, Seifert B, Arras M. Burrowing behavior as an indicator of post-laparotomy pain in mice. Front Behav Neurosci. 2010;4:165.

34 Rutten K, Schiene K, Robens A, Leipelt A, Pasqualon T, Read SJ, et al. Burrowing as a non-reflex behavioural readout for analgesic action In a rat model of sub-chronic knee joint inflammation. Eur J Pain. 2014;18(2): 204-12.

35 Whittaker AL, Lymn KA, Nicholson A, Howarth GS. The assessment of general well-being using spontaneous burrowing behaviour in a short-term model of chemotherapy-induced mucositis in the rat. Lab Anim. 2015;49(1): 30-9.

36 Lang A, Schulz A, Ellinghaus A, SchmidtBleek K. Osteotomy models - the current status on pain scoring and management in small rodents. Lab Anim. 2016;50(6):433-41.

37 Langford DJ, Bailey AL, Chanda ML, Clarke SE, Drummond TE, Echols S, et al. Coding of facial expressions of pain in the laboratory mouse. Nat Methods. 2010;7(6):447-9.
38 Sotocinal SG, Sorge RE, Zaloum A, Tuttle AH, Martin LJ, Wieskopf JS, et al. The rat grimace scale: a partially automated method for quantifying pain in the laboratory rat via facial expressions. Mol Pain. 2011;7:55.

39 Burkholder T, Foltz C, Karlsson E, Linton CG, Smith JM. Health evaluation of experimental laboratory mice. Curr Protoc Mouse Biol. 2012;2:145-65.

40 Alam M, Schwabe K, Krauss JK. The pedunculopontine nucleus area: critical evaluation of interspecies differences relevant for its use as a target for deep brain stimulation. Brain. 2011;134(Pt 1):11-23.

41 Voges J, Krauss JK. Neurochirurgische und technische aspekte der tiefen hirnstimulation. Nervenarzt. 2010;81(6):702-10.

42 Lozano AM, Lipsman N, Bergman H, Brown P, Chabardes S, Chang JW, et al. Deep brain stimulation: current challenges and future directions. Nat Rev Neurol. 2019;15(3):148-60.

43 Choleris E, Thomas AW, Kavaliers M, Prato FS. A detailed ethological analysis of the mouse open field test: effects of diazepam, chlordiazepoxide and an extremely low frequency pulsed magnetic field. Neurosci Biobehav Rev. 2001;25(3):235-60.

44 Flecknell PA, Roughan JV. Assessing pain in animals - putting research into practice. Anim Welf. 2004;13:71-5.

45 Sneddon LU, Elwood RW, Adamo SA, Leach MC. Defining and assessing animal pain. Anim Behav. 2014;97:201-12.

46 Graf R, Cinelli P, Arras M. Morbidity scoring after abdominal surgery. Lab Anim. 2016; 50(6):453-8.

47 Sousa N, Almeida OF, Wotjak CT. A hitchhiker's guide to behavioral analysis in laboratory rodents. Genes Brain Behav. 2006;5 Suppl 2:5-24.

48 mytalbot/RELSA: relative severity assessment score for laboratory animals. 2020 [cited 2020 Mar 23]. Available from: https://rdrr.io/ github/mytalbot/RELSA/man/relsa.html. 